\title{
Implicit Skolemization: Efficient Reference to Dependent Entities
}

\author{
Lenhart Schubert \\ Department of Computer Science \\ University of Rochester \\ Rochester, NY 14627-0226, USA \\ schubert@cs.rochester.edu
}

\section{Introduction}

Consider the following examples of functional reference.

(1) All of the graduates received a job offer (at the job fair), and all of them accepted their offer.

(2) If all of the graduates received a job offer then all of them accepted their offer.

While these examples resemble the kinds of sentences that have motivated theories of dynamic binding such as DRT and DPL [10], they are not easily handled within these standard frameworks. The problem is that the entity referred to by "their offer" is variable. The logical form (LF) of (1) is not captured by

(3) $(\forall \mathrm{x})$ [graduate $(\mathrm{x}) \rightarrow(\exists \mathrm{y})$ job-offer $(\mathrm{y}) \wedge \operatorname{receive}(\mathrm{x}, \mathrm{y})] \wedge$

$(\forall \mathrm{x})[\operatorname{graduate}(\mathrm{x}) \rightarrow \operatorname{accept}(\mathrm{x}, \mathrm{y})]$

since the final occurrence of $y$ is not dynamically bound - the values assigned to $y$ in the existential clause do not persist beyond the scope of the initial $\forall$-quantifier. The following LF does capture the meaning of (1):

(4) $(\forall \mathrm{x})[\operatorname{graduate}(\mathrm{x}) \rightarrow(\exists \mathrm{y})$ job-offer $(\mathrm{y}) \wedge \operatorname{receive}(\mathrm{x}, \mathrm{y})] \wedge$ $(\forall \mathrm{x})[\operatorname{graduate}(\mathrm{x}) \wedge(\exists \mathrm{y})($ job-offer $(\mathrm{y}) \wedge \operatorname{receive}(\mathrm{x}, \mathrm{y}))] \rightarrow \operatorname{accept}(\mathrm{x}, \mathrm{y})$.

Here the final occurrence of $y$ is dynamically bound by the existentially quantified wff in the antecedent, and the binding evidently depends on the graduate $x$ under consideration, as required. However, this putative LF bears no simple, systematic relation to (1); in essence, it repeats the content of the first half of (3) in order to provide an appropriate dynamic binding environment for $y$ in the second half. So from a computational semantics perspective, this is not an attractive approach.

We propose a systematic alternative requiring no ad hoc repetition of material. The idea is to generalize the state-change mechanism of DPL so that what is 'carried forward' as a value of $y$ from a formula like that in the first half of (3) is a function - in this case, one that maps graduates to their job offers. This function can then be applied in the second half of (3) to pick out job offers corresponding to particular graduates; i.e, the final phrase in (3) becomes accept $(x, y(x))$. We will indicate that this way of treating the LFs of functionally dependent anaphors is applicable as well to many instances of bridging anaphora. Finally we will briefly comment on the utility of our generalization of DPL in representing definite reference to functionally dependent entities of the sort encountered in frames, scripts, and generic sentences. 


\section{Generalized variable assignments and functional DPL}

The consistent use of existentially quantified variables as functions entails some slight changes in ordinary logical syntax. In particular, if a variable is bound anywhere by an existential quantifier, it may not be bound anywhere else by an existential or universal quantifier. Instead, any occurrences outside the scope of its $\exists$-quantifier should involve application of the variable to 0 or more arguments, thus yielding a term. In effect, existential variables will serve outside their 'defining contexts' as implicit Skolem constants and functions. More precisely, suppose that a formula $(\forall x) \phi$ contains a formula $(\exists y) \psi$ in its scope. We say that $y$ depends on $x$ if $(\exists y) \psi$ does not lie within the scope of a negation in $\phi$. Then the 'defining context' (relative to a formula $\Omega$ ) of $y$ in a formula $(\exists y) \psi$ (embedded by $\Omega$ ) is $(\exists y) \psi$ itself if $y$ does not depend on any $\forall$-variables (in $\Omega$ ), and otherwise it is the largest formula of form $(\forall x) \phi$ (in $\Omega$ ) such that $y$ depends on $x$. The adicity of an existential variable $y$ used outside the scope of its $\exists$-quantifier is then the number of $\forall$-variables on which it depends in its defining context, relative to the smallest formula $\Omega$ that contains both the $\exists$-quantifier of $y$ and the external occurrence of $y$. However, rather than insisting on conformity with this subtle constraint, we will formulate the semantics of predication so that atoms containing 'ill-formed' terms will be treated as false.

Given a vocabulary $\operatorname{Var}$ of variables and a domain of interpretation $\mathcal{D}$, a generalized variable assignment (gva) is any partial function ${ }^{1}$

$$
\begin{aligned}
& U: \operatorname{Var} \rightarrow \mathcal{F}, \text { where } \\
& \mathcal{F}=\bigcup_{n \in \mathcal{N}} \mathcal{F}_{n}, \text { and } \\
& \begin{aligned}
\mathcal{F}_{n} & =\mathcal{D}^{n} \rightarrow \mathcal{D} \\
& =\mathcal{D} \rightarrow(\mathcal{D} \rightarrow(\ldots(\mathcal{D} \rightarrow \mathcal{D}) \ldots)),
\end{aligned}
\end{aligned}
$$

( $n$ arrows), the class of (curried) partial functions from $\mathcal{D}^{n}$ to $\mathcal{D}$, where $\mathcal{F}_{0}$ is just $\mathcal{D}$. In the following, all occurrences of $U$ and $V$ (with or without subscripts) denote gva's. $U_{y: f}$, for any $y \in \operatorname{Var}$ and any individual or function $f \in \mathcal{F}$, will denote the variant of $U$ which is the same as $U$ except that its value at $y$ is $f . \mathcal{M}=(\mathcal{D}, \mathcal{I})$, where $\mathcal{I}$ is an ordinary interpretation function.

Much as in DPL, the semantic value of a formula $\phi$ relative to a model $\mathcal{M}$, written $\llbracket \phi]_{\mathcal{M}}$, will be a satisfaction set whose elements are pairs $\langle U, V\rangle$ of gva's. If $V=U$ for all such elements, $\phi$ is said to be $\mathcal{M}$-static, and otherwise it is $\mathcal{M}$-dynamic. ${ }^{2}$ If $\phi$ is $\mathcal{M}$-static for all models $\mathcal{M}$, it is (uniformly) static, and otherwise it is (potentially) dynamic. Formulas containing no $\exists$-quantifiers and ones whose $\exists$-quantifiers are embedded by negations turn out to be uniformly static, as in DPL. However, certain formulas that are uniformly static in DPL, such as $(\forall x)[Q(x) \rightarrow(\exists y) P(y)]$ or $Q(x) \vee(\exists y) P(y)$, may be $\mathcal{M}$-static or $\mathcal{M}$-dynamic in our semantics, depending on $\mathcal{M}$; in particular, both formulas are $\mathcal{M}$-static if $\mathcal{I}(P)=\emptyset$ and $\mathcal{M}$-dynamic otherwise. We will consider some more meaningful examples after filling in a few semantic details below. A useful related notion is the following: a variable $y \in \operatorname{Var}$ is $\mathcal{M}$-static in $\phi$ if $V(y)=U(y)$ for all $\langle U, V\rangle \in \llbracket \phi]_{\mathcal{M}}$; otherwise it is $\mathcal{M}$-dynamic in $\phi ; y$ is (uniformly) static in $\phi$ if it is $\mathcal{M}$-static in $\phi$ for all models $\mathcal{M}$, and it is (potentially) dynamic in $\phi$ otherwise. Roughly speaking, stativity provides a semantic criterion for distinguishing variables that are free or $\forall$-bound in $\phi$ from those that are $\exists$-bound in $\phi$, except that $\exists$-variables whose quantifier occurs within a static formula are themselves static. (Again,

\footnotetext{
${ }^{1}$ In a preliminary ESSLLI'04 version of this paper, the author used total functions [19]; the use of partial functions allows some subtle problems to be addressed, and enables a fuller treatment of generic sentences.

${ }^{2}$ Static formulas are often termed conditions, but this terminology makes it awkward, for instance, to talk about the conditions under which a formula is a 'condition'.
} 
occurrences within the scope of $\neg$, or the occurrences of $(\exists y) P(y)$ in the two formulas above, with $\mathcal{I}(P)=\emptyset$, illustrate this.)

The semantics of terms, predication, equality, negation and conjunction are identical to those of DPL, modulo the use of gva's. Thus predication, equality, and negation are necessarily static, while conjunctions may be dynamic. One minor wrinkle is that in order to deal with undefined values of partial functions, and to render adicity violations in the use of variables as functors harmless, we assume that a term $\sigma(\tau)$ lacks a value (relative to a model and a gva) if $\sigma$ lacks a value or denotes an individual, or $\tau$ lacks a value or denotes a function. The usual semantics of predication will then make atoms containing valueless terms false, assuming that a tuple with undefined elements cannot be a member of a set, and that $\llbracket \sigma=\tau]_{\mathcal{M}}$ is reckoned as false if either or both terms are undefined. (By "false" we mean here that a gva $U$ that renders a term valueless relative to the given model cannot give rise to an element $\langle U, V\rangle$ in the satisfaction set of the atom containing that term.)

The intuitive idea in the following semantics of quantification is this. We want a set of $n$ nested universally quantified sentences, all embedding an existentially quantified sentence $(\exists y) \phi$ to 'output' an $n$-place function as the value of $y$, at least when $y$ is not static in the scopes of the $\forall$-quantifiers. The semantics of universal quantification relies on our semantic criterion for 'detecting' nonstatic $\exists$-variables, using it to assign a function as value of any such existential variable in its scope, in each 'output' assignment it produces. The function is chosen so that it 'works' uniformly for all values of the universally quantified variable. The correct adicity of these functions is obtained through the use of curried functions, i.e., each level of universal quantification embedding an existential adds another level of functional dependence.

(5) $\left.\llbracket(\exists y) \phi]_{\mathcal{M}}=\left\{\langle U, V\rangle \mid \text { for some } d \in \mathcal{D},\left\langle U_{y: d}, V\right\rangle \in \llbracket \phi\right]_{\mathcal{M}}\right\} ;$

(6) $\llbracket(\forall x) \phi \rrbracket]_{\mathcal{M}}=\left\{\langle U, V\rangle \mid \text { for all } d \in \mathcal{D} \text {, there is a gva } V^{\prime} \text { such that }\left\langle U_{x: d}, V_{x: d}^{\prime}\right\rangle \in \llbracket \phi\right]_{\mathcal{M}}$, where for all variables $y, V^{\prime}(y)=V(y)=U(y)$ if $y$ is $\mathcal{M}$-static in $\phi$, and $V^{\prime}(y)=V(y)(d)$ if $y$ is $\mathcal{M}$-dynamic in $\left.\phi\right\}$.

(We assume that truth of $V^{\prime}(y)=V(y)(d)$ requires $V(y)$ to be a function, i.e., an element of $\mathcal{F} \backslash \mathcal{D}$, even if $V^{\prime}(y)$ is undefined - in which case $V(y)$ must be undefined at $d .^{3}$ ) Note how (5) and (6) formalize the above intuitions: in (6), because of the final clause, a function $V(y)$ may be returned (in the 'output' state of $(\forall x) \phi$ ) as the denotation of a variable $y$ that is $\exists$-quantified within $\phi$, provided that the values $V(y)(d)$ of that function for all $d \in \mathcal{D}$ may be produced as values of $y$ in the 'output' state of $\phi$, i.e., as values of $V^{\prime}(y)$. In turn, (5) ensures (as in standard DPL) that values produced for $y$ are ones that verify the scope of the $\exists$-quantifier.

It is also clear from the definition of static/dynamic variables that (6) allows only variables $\exists$-quantified within $\phi$ to export (new) functional output values. We have already noted that not all such $\exists$-quantified variables export (new) functional output values. An intuitively meaningful example is the following:

$(\forall \mathrm{x})[\operatorname{mule}(\mathrm{x}) \rightarrow \neg(\exists \mathrm{y})$ offspring $(\mathrm{y}, \mathrm{x})]$,

Here $y$ receives no (new) value since all negated formulas are static by definition. Likewise no functional value (distinct from the input value) is exported for $y$ in

\footnotetext{
${ }^{3}$ To see that $V^{\prime}(y)$ can be undefined, even when $y$ is $\mathcal{M}$-dynamic in $\phi$, consider for instance $\phi=Q(x) \vee$ $(\exists y) P(x, y)$, where $Q(x)$ is true in $\mathcal{M}$ for a certain value of $x$, say $d$, and $(\exists y) P(x, y)$ is true for other values (and for all values of $x$, at least one of the disjuncts is true). Then we can have $\left.\left\langle U_{x: d}, U_{x: d}\right\rangle \in \llbracket \phi\right]_{\mathcal{M}}$, thanks to the first disjunct, even if $U(y)$ is undefined.
} 
$(\forall \mathrm{x})[\operatorname{unicorn}(\mathrm{x}) \rightarrow(\exists \mathrm{y})$ magical-horn $(\mathrm{y}, \mathrm{x})]$

as long as there are no unicorns in the model under consideration, since a conditional with a false antecedent is static (see below). If the model allows for unicorns but not magical horns, then of course the satisfaction set of the conditional is empty and so certainly no values are exported for $y$ or for any other variable. The following "Implicit Skolemization" theorem formalizes and generalizes the above observations.

Theorem (Implicit Skolemization). For any formula $\phi$ and model $\mathcal{M}$, if $\langle U, V\rangle \in \llbracket \phi]_{\mathcal{M}}$ then for any variable $y$ that is $\mathcal{M}$-dynamic in $\phi$, $\phi$ contains a (unique) $\exists$-quantifier binding $y$, where that quantifier is not embedded by any negation in $\phi$, and $V(y) \in \mathcal{F}_{n}$ where $n$ is the number of $\forall$-quantifiers in $\phi$ embedding the $\exists$-quantifier for $y$.

Proof: See Appendix. The proof of course depends on the completion of the definition of $\llbracket \cdot]_{\mathcal{M}}$, which follows.

Disjunction and conditionals do not behave as in standard DPL: they can 'export' dynamic bindings of existential variables. At least for conditional sentences this is essential to our enterprise, since otherwise universally quantified contexts whose scope is a conditional sentence (the usual case - see (3)) would fail to export functional values for existentials lying within those conditionals:

(7) $\llbracket \phi \vee \psi \rrbracket_{\mathcal{M}}=\{\langle U, V\rangle \mid \text { either }\langle U, V\rangle \in \llbracket \phi]_{\mathcal{M}}$ or $\left.\left.\langle U, V\rangle \in \llbracket \psi\right]_{\mathcal{M}}\right\}$

(8) $\llbracket \phi \rightarrow \psi]_{\mathcal{M}}=\left\{\langle U, V\rangle \mid\right.$ either $V=U$ and for no gva $U^{\prime},\left\langle U, U^{\prime}\right\rangle \in \llbracket \phi \rrbracket_{\mathcal{M}}$, or for some gva $\left.U^{\prime},\left\langle U, U^{\prime}\right\rangle \in \llbracket \phi\right]_{\mathcal{M}}$ and $\left.\left.\left\langle U^{\prime}, V\right\rangle \in \llbracket \psi\right]_{\mathcal{M}}\right\}$

(Note that we could have written the second alternative on the right-hand side of (8) as $\left.\langle U, V\rangle \in \llbracket(\phi \wedge \psi)]_{\mathcal{M}}.\right)$ So for instance the anaphoric " $i t$ " in the following sentences could be translated in terms of dynamically bound variables in the LFs (imagine a euphoric physicist uttering (9), and a somewhat verbose robber uttering (10)):

(9) Either I've lost my mind, or I've come up with a Theory of Everything. It combines ideas from string theory, holographic-universe theory, and loop quantum gravity.

(10) If you value your life, you'll hand over some cash fast. It had better be more than a few bucks.

(11) Holmes reasoned that if an intruder had opened the safe, he must have left fingerprints on the knobs of the safe. ?And indeed he had.

While anaphoric reference to an indefinite within a disjunction or conditional is typically infelicitous as a result of Gricean implicatures, there seems to be no particular advantage to blocking such reference in the target logic for semantic representation. In fact, when the implicatures are blocked (as in (9), where one presumes the falsity of the first disjunct, or in (10), where one presumes the truth of the antecedent of the conditional), we want dynamic binding to be enabled. In a sense, the proposed semantics 'explains' why an attempted reference to an indefinite $(\exists y) \phi$ occurring within a disjunction or conditional is typically infelicitous: if nothing can be presumed about the truth of the immediate constituents of such a sentence, then even if the sentence as a whole is true, an external anaphor $y$ may fail to refer to anything pertinent (or anything at all). ${ }^{4}$

\footnotetext{
${ }^{4}$ It can be argued that we may want to refer into doubly negated contexts as well, in view of examples such as "It is not true that this house doesn't have a bathroom. It is in the attic." This point can be generalized to
} 
In addition, (8) differs from standard DPL in that it presupposes a 'weak' interpretation of conditionals. For example, it renders the sentence

If John received a job offer today, then he accepted it

true in circumstances where John received multiple job offers but accepted just one; whereas on a 'strong' reading, the sentence would be false. While it would be possible to change the semantics to deliver strong readings (while still allowing export of variable bindings from conditionals), we take weak readings to be basic (as for instance in [6] and [18]). ${ }^{5}$

We will also have occasion in section 3.1 to consider the following 'maximally dynamic' version of (7), in order to deal with referential difficulties posed by quantified sentences with a disjunctive restrictor:

$\left.\left(7^{\prime}\right) \llbracket \phi \vee \psi\right]_{\mathcal{M}}=\{\langle U, V\rangle \mid \text { (a) }\langle U, V\rangle \in \llbracket \phi]_{\mathcal{M}}$ and for no $U^{\prime},\left\langle U, U^{\prime}\right\rangle \in \llbracket \psi \rrbracket_{\mathcal{M}}$; or (b) $\langle U, V\rangle \in \llbracket \psi \rrbracket_{\mathcal{M}}$ and for no $U^{\prime},\left\langle U, U^{\prime}\right\rangle \in \llbracket \phi \rrbracket_{\mathcal{M}}$; or (c) $\left.\langle U, V\rangle \in \llbracket(\phi \wedge \psi) \rrbracket_{\mathcal{M}}\right\}$

For this semantics, reference will be successful in (9) even if the speaker has, in fact, lost his mind, as long as he has also come up with a Theory of Everything. (Note that by the second part of condition $\left(7^{\prime}\right)(\mathrm{a})$, no pair $\langle U, V\rangle$ that verifies the first disjunct alone can verify the disjunction, if the conjunction is true.) Likewise reference will be successful in the following example, even if both disjuncts are true:

(12) Either John has a very bad violin, or he plays it very poorly.

The definitions of truth and entailment are as follows, for formulas $\phi$ and $\psi$.

(13) $\phi$ is true [false] in model $\mathcal{M}$ relative to gva $U$ iff for some [no] gva $V,\langle U, V\rangle \in \llbracket \phi]]_{\mathcal{M}}$.

(14) $\phi \models \psi$ iff for all models $\mathcal{M}$ and all $U, V$, if $\langle U, V\rangle \in \llbracket \phi]_{\mathcal{M}}$ then for some gva's $V^{\prime}, W$, $\left\langle U, V^{\prime}\right\rangle \in \llbracket \phi \rrbracket_{\mathcal{M}}$ and $\left.\left\langle V^{\prime}, W\right\rangle \in \llbracket \psi\right]_{\mathcal{M}}$.

(14) aligns entailment with the conditional semantics (8), in the sense that it supports the equivalence

(15) $\phi \models \psi$ iff $=\phi \rightarrow \psi$.

This is easily proved in both directions from (13) and (14).

(14) also allows for the following sort of detachment:

(16) $[(\exists x P(x)) \rightarrow Q(x)], \exists y P(y) \models Q(x)$,

where the premises are ordered as shown.

It is also useful, for application to linguistic semantics, to define a notion of truth for a text, viewed as a nonempty sequence of formulas. This notion of truth is not relativized to a gva:

(17) Text $\phi_{1}, \ldots, \phi_{n}$ is true [false] in model $\mathcal{M}$ iff for some [no] gva $V,\langle\emptyset, V\rangle \in \llbracket\left(\phi_{1} \wedge \ldots \wedge\right.$ $\left.\left.\phi_{n}\right)\right]_{\mathcal{M}}$.

locutions creating downward-entailing contexts, such as the object positions of deny and lack in "I deny that this house lacks a bathroom. It is in the attic." However, allowing for this in the proposed semantics appears to require adding negative satisfaction sets (bearing witness to the falsity of a sentence) to the semantic machinery, a significant complication that we do not pursue here.

${ }^{5}$ Geurts [9] suggests that subjects confronted with the task of judging the truth of donkey sentences for given states of affairs (e.g., presented visually) don't actually discern and choose between separate 'readings'; rather, they use the form of the sentence, certain intuitions about individuation (i.e., how hard or easy it is to 'count' an individual more than once in evaluating multiple situations), and weak uniqueness implicatures to arrive at a truth value judgement directly. His view seems reconcilable with the position taken here. For instance, the supposed strong reading of "Every student who received a job offer turned it down" can be regarded as the result of counting students with multiple job offers multiple times, once for each job offer thereby preserving the uniqueness presumption that there is just one job offer per student (as offeree). 
In other words, we start out with the empty assignment, and allow this to be dynamically extended by existential quantifiers (and by universal quantifiers within their scopes). This notion of truth will be relevant to some examples discussed in the following section. Note that under this definition, since we are "filling in" undefinedness with falsity, a text consisting of (or containing as sequence element, with no prior occurrence of $x$ ) an open sentence such as $P(x)$ is simply false in all models. However, we count a formula as logically false only if it is false in all models relative to all gva's, not just the empty gva. The presumption is that truth/falsity of texts is of interest chiefly for dynamically closed texts, i.e., ones in which all variables receive values either through local binding by a quantifier or through dynamic binding via a prior existentially quantified formula.

\section{Some applications}

The semantics just outlined allows for a very simple, direct logical representation of (disambiguated) referential connections in ordinary discourse, including not only the familiar types of donkey anaphora, but also anaphora involving functional dependencies, as in (1) and (2). (We will shortly delve into some subtleties in the use of such functional terms.) The present proposal is related to the author's scheme of dynamic Skolemization [18], but deals satisfactorily with negative environments such as "It is not the case that John owns a donkey and beats it" (which under dynamic Skolemization is true if John owns two donkeys and beats only one). ${ }^{6}$

In the remainder of this discussion, we consider three issues: the appropriate use of functional expressions as logical forms of anaphoric noun phrases; bridging anaphora; and frame/script-like knowledge.

\subsection{Using functional expressions for anaphoric noun phrases}

Ordinary language typically quantifies over restricted nominal domains (rather than over the entire domain of individuals), so to facilitate the discussion of LFs for functional anaphora we will generalize (6) to allow for a restrictor. We could have simply relied on the equivalence

$(\forall x: \phi) \psi \Leftrightarrow(\forall x)(\phi \rightarrow \psi)$

but we wish to set the stage as well for a discussion of quantifiers like Most, for which no such equivalence is available.

For convenience we define the notion of a truth domain, for any variable $x$, formula $\phi$, model $\mathcal{M}$, and gva $U$ as follows:

(18) $\left.T_{\mathcal{M}, U}(x, \phi)=_{\text {def }}\left\{d \in \mathcal{D} \mid \text { for some gva } V,\left\langle U_{x: d}, V\right\rangle \in \llbracket \phi\right]_{\mathcal{M}}\right\}$

We now generalize (6) to

(19) $\llbracket(\forall x: \phi) \psi \rrbracket_{\mathcal{M}}=\left\{\langle U, V\rangle \mid\right.$ (a) $T_{\mathcal{M}, U}(x, \phi)=\emptyset$ and $V=U$, or else

(b) $T_{\mathcal{M}, U}(x, \phi) \neq \emptyset$ and for all $d \in \mathcal{D}$, there is a gva $V^{\prime}$ such that

(i) if $d \in T_{\mathcal{M}, U}(x, \phi)$ then $\left.\left\langle U_{x: d}, V_{x: d}^{\prime}\right\rangle \in \llbracket \phi \wedge \psi\right]_{\mathcal{M}}$ and otherwise $V^{\prime}=U$; and

(ii) for all variables $y, V^{\prime}(y)=V(y)=U(y)$ if $y$ is $\mathcal{M}$-static in $(\phi \wedge \psi)$, and

$V^{\prime}(y)=V(y)(d)$ if $y$ is $\mathcal{M}$-dynamic in $\left.(\phi \wedge \psi)\right\}$.

We should observe, first of all, that this definition is indeed semantically equivalent to $(\forall x)(\phi \rightarrow \psi)$ :

Proposition 1. For all models $\left.\mathcal{M}, \llbracket(\forall x: \phi) \psi]_{\mathcal{M}}=\llbracket(\forall x)(\phi \rightarrow \psi)\right]_{\mathcal{M}}$.

Proof: See Appendix.

\footnotetext{
${ }^{6}$ The idea of treating variables as functions is suggested as an alternative to dynamic Skolemization at the end of that paper.
} 
Note that according to condition (a), when the restrictor truth domain is empty, $V(y)$ is the same as $U(y)$ for all variables $y$. For example, as we observed previously for the sentence "Every unicorn $x$ has a magical horn $y$ ", if our model has no unicorns, then a subsequent term such as $y$ (Indigo) will refer to something arbitrary or will fail to refer (leading to falsity of the embedding predication). This is a reasonable outcome under the assumed circumstances.

Note also that for $d$ outside $T_{\mathcal{M}, U}(x, \phi)$, 'old' values $U(y)$ of variables are preserved by $V$, since in that case $V(y)(d)$ in (b)(ii) equals $U(y)$, by the 'otherwise'-clause in (b)(i). So in particular undefinedness is preserved in the application of the notion of textual truth (17). Thus if we consider a restrictor $\phi$ that is true relative to some $x$-variants $U_{x: d}$ of $U$ and false relative to others, then $V(y)(d)$ will be $U(y)$ (which can be arbitrary, or undefined) for those $d \in \mathcal{D}$ that fail to verify $\phi$ relative to $U_{x: d}$.

However, these spurious or missing values are irrelevant to uses of a term such as $y(z)$ as the LF of an anaphor, as long as in the context of use, $z$ is guaranteed to satisfy the 'applicability conditions' for $y$, i.e., the conditions $C$ such that the defining context for $y$ could be rewritten as $(\forall x: C(x))(\exists y)[\ldots]$. (More generally, there may be nested quantifiers, with applicability conditions contributed by each level of $\forall$-quantification.) For example, in the following sentence,

(20) Every student $x$ wrote a paper $y$, but no students $x$ who took the exam handed in their paper on time,

$y$ would be assigned a function whose value for any student is a paper written by that student, while its value for other arguments would be arbitrary or undefined. In other words, the applicability condition for $y$ is that the value of the argument of $y$ must be a student. This certainly holds for students who took the exam, and so $y(x)$ can be properly used as the LF of "their paper". On the other hand, use of such an LF would violate the suggested applicability conditions in the sentence

(21) Every student $x$ wrote a paper $y$ or took the exam, but no students $x$ handed in their paper on time. ${ }^{7}$

Here the applicability condition for $y$ is that its argument should denote a student who didn't take the exam, since the defining context for $y$ is equivalent to "Every student $x$ who didn't take the exam wrote a paper $y$ "; but the quantification "no students $x$..." iterates over all students, including those who took the exam, and for these students, $y(x)$ may 'accidentally' denote some entity (such as an exam booklet) that was indeed handed in (on time) by $x$; thus "no students $x$ handed in $y(x)$ on time" may well be formally false even when "no students $x$ handed in their paper on time" is intuitively true.

However, if we rigidly enforce applicability conditions we are left with the problem of assigning an LF to anaphors like that in (21). While the use of "their paper" in (21) does seem to falsely presuppose or at least implicate that every student has a paper, the sentence seems true even if that presupposition or implicature is violated, as long as none of the students who wrote a paper handed it in on time. A possible answer is that $y(x)$ provides the correct interpretation when we employ the 'maximally dynamic' disjunction semantics $\left(7^{\prime}\right)$ rather than (7), and bring to bear our notion of textual truth (as per (17)), rather than truth relative to an arbitrary assignment. Observe that if (21) is treated as a text and our initial assignment is empty, then for any assignment $V$ that can result from the first clause,

\footnotetext{
${ }^{7}(21)$ was suggested by Ken Shan (personal communication); its variant (20) and the discussion of these sentences were motivated by his comments
} 
$V(y)(d)$ will be well-defined for all and only the students $d$ who wrote a paper, where that paper is given by $V(y)(d)$. Thus if we formalize "their paper" as $y(x)$ in the second clause, this will have a reference precisely for those students who wrote a paper, and the desired claim will be made about these students. For students who did not write a paper, the value of $y(x)$ will be undefined, but the claim that they did not hand in $y(x)$ will be true, as an undefined entity cannot be handed in, according to our semantics of predication. If "but $n o$ " in (21) were replaced by "and all', then truth of the text would indeed require that all students wrote and handed in a paper. This seems intuitively reasonable - cf., David Ahn's discussion of the sentence "Every German loves his kangaroo" in [1].

\subsection{Bridging anaphora}

The phenomena we have been focusing on appear in a slightly different guise in bridging anaphora, illustrated in (22).

(22) Cora walked up to a house. She knocked on the door.

First, we want to point out the simplicity of the logical form of the second sentence on a functional analysis. Suppose that part of the background knowledge for (22) is

(23) $(\forall \mathrm{x})$ house $(\mathrm{x}) \rightarrow(\exists \mathrm{y}) \operatorname{door}(\mathrm{y}) \wedge$

$$
\text { part-of }(\mathrm{y}, \mathrm{x}) \wedge \text { at-front-of }(\mathrm{y}, \mathrm{x})]
$$

(Every house has a front door). On our semantics, this makes available a function, namely $y$, for referring to the door of a house. Thus the (resolved) LF of the second sentence of (22) becomes (ignoring tense)

(24) knock-on(Cora,y(z)),

where $z$ is the $\exists$-variable for the house in the LF of the first sentence.

This LF also has significant semantic advantages: while $y(z)$ is a specific choice of referent for "the door" (viz., the front door), it does not commit us to a presumption of uniqueness, in contrast with a more 'literal' interpretation of the definite such as $\imath w[\operatorname{door}(w) \wedge$ part$o f(w, z)]$. A uniqueness assumption may well be incorrect; (22) is perfectly felicitous even if houses are also known to have back doors, or, occasionally, multiple front doors. ${ }^{8}$

\subsection{Frames, scripts, and generic sentences}

In AI, general knowledge of the sort exemplified by (23) has traditionally been represented using frames [13] - packets of knowledge about particular kinds of things (such as a house) belonging to an inheritance hierarchy (perhaps including buildings, architectural objects, or artifacts more generally), and providing information about various parts or aspects of those kinds of things (such as walls, doors, windows, location, etc.) via 'slots' interpreted as functions. Over time, frames have evolved into description logics [3], but the idea remains much the same. Closely related to frames are scripts [15], except that these attempt to formalize familiar kinds of structured events (such as dining at a restaurant) rather than structured objects. But just as in the case of frames, the relevant aspects (i.e, participants, and subevents, such as entering, getting seated, ordering, etc.) can be modelled as entities ('roles') functionally dependent on the whole, and thus frame or description logic formalisms can be applied.

While well-studied from a logical and computational perspective, frames and description logics have been developed more or less independently of linguistic considerations. In particular, there has been little discussion of the relation between knowledge expressed in

\footnotetext{
${ }^{8}$ This of course means that $y(z)$ may not be the only possible choice of referent, but that is an issue that any reference resolution strategy must face. Similar examples are "He reached into his pocket", "He likes his neighbor, "He went to the doctor", etc.
} 
language and knowledge expressed in frames or description logics. Knowledge engineers who employ the latter simply use their intuitions and their understanding of the target formalisms to code knowledge about the domain of interest. But one would expect that such work would become much easier if the knowledge to be formalized could be expressed in ordinary language and automatically translated into a formal representation. Indeed, much knowledge coding could be avoided altogether if the general knowledge that can be found in on-line lexicons, encyclopedias, manuals, and other text corpora could be automatically interpreted and extracted.

What is potentially attractive about the present proposal (and this is something it shares with dynamic Skolemization) is that it shows how the functional dependencies expressed by slots in frames and roles in scripts (and slots or attributes in description logics) might be directly derived from linguistically expressed general knowledge. For example, an LF similar to (23) might be the logical translation of an ordinary sentence (something like "Every house has [as part of it] a door at its front"), and this translation provides a "frontdoor function' $y$ that can subsequently be used outside its defining context, just like a slot or role name, to refer to a particular aspect - the front door - of instances (or subtypes) of the general type, house.

In the case of structured events, we would similarly obtain functions for referring to participants or subevents simply by $\exists$-quantification. For example, the LF of a verbal description of the events that transpire when a person dines at a restaurant would probably involve, among other things, an $\exists$-quantified entering event, an $\exists$-quantified getting-seated event, an $\exists$-quantified server, and so on. (See [18] for a simple verbally expressed 'dining' script and the interpretation of its roles/subevents as functions.) Since the $\exists$-quantifiers lie within the scope of a $\forall$-quantifier over dining events (and perhaps patrons and restaurants), they make available functions that can subsequently be used to refer to roles and subevents in specific dining events (or special cases of such events).

One advantage of using 'ordinary logic' (adapted as suggested here, and perhaps enriched in other ways) rather than frames, scripts or description logics as the target language for linguistically expressible knowledge is that we gain flexibility and expressiveness. For example, the fact that chimneys of houses serve to vent combustion gases from furnaces or fireplaces would be virtually inexpressible in description logics developed so far. ${ }^{9}$ It would also be hard to integrate, say, a frame for a dining establishment with a script for dining - allowing for such facts as that tables for patrons are placed in close proximity to one another, and that servers serve patrons at multiple tables.

Our proposals here also seem extensible in a way that would meet the referential requirements of general knowledge admitting exceptions, i.e., generic knowledge. In keeping with the literature on generics (e.g., $[5,2]$ ), let us assume the availability of generalized quantifiers such as Most and Few. The semantics of Most would be given by the following analogue of (19), with "most" interpreted in some specific way (say, more than half, in the case of finite sets).

(25) $\llbracket($ Most $x: \phi) \psi]_{\mathcal{M}}=\left\{\langle U, V\rangle \mid\right.$ (a) $T_{\mathcal{M}, U}(x, \phi)=\emptyset$ and $V=U$, or else (b) $T_{\mathcal{M}, U}(x, \phi) \neq \emptyset$ and most of its elements are contained in $T_{\mathcal{M}, U}(x, \phi \wedge \psi)$, and for all $d \in \mathcal{D}$, there is a gva $V^{\prime}$ such that

(i) if $d \in T_{\mathcal{M}, U}(x, \phi \wedge \psi)$ then $\left.\left\langle U_{x: d}, V_{x: d}^{\prime}\right\rangle \in \llbracket \phi \wedge \psi\right]_{\mathcal{M}}$ and otherwise $V^{\prime}=U$; and (ii) for all variables $y, V^{\prime}(y)=V(y)=U(y)$ if $y$ is $\mathcal{M}$-static in $(\phi \wedge \psi)$, and

\footnotetext{
${ }^{9}$ Well, we could have a PURPOSE slot with value VENT-COMBUSTION-GASES-FROM... etc., but that would hardly support useful inferential linkages to notions like 'venting', 'combustion', etc.
} 


$$
\left.V^{\prime}(y)=V(y)(d) \text { if } y \text { is } \mathcal{M} \text {-dynamic in }(\phi \wedge \psi)\right\} \text {. }
$$

One point to note about this definition is that the implicit Skolem functions that are generated by existentials within the restrictor or nuclear scope provide meaningful values corresponding not only to most elements of the restrictor domain, but to all elements for which the restrictor and nuclear scope are true - which potentially includes the entire restrictor domain (namely, in the case where 'most' could truthfully be strenghtened to 'all').

Now, one thing we can express with such an extension of our language is generic reference to dependent entities whose defining context is universal. For example, consider again the front-door function $y$ 'generated' in (23) (its defining context); we could express that the front door of a house usually (in most instances) opens inward as

(26) (Most x: house(x))opens-into(y(x),x).

(26) is much simpler (and arguably more easily obtainable from surface form) than DRTor DPL-based LFs, which would require 'copying-over' of the defining properties of $y$ from (26) into the restrictor of Most in (26).

Further, suppose that the defining context for a dependent entity like the front door in (23) is itself generic, i.e., it states that most (rather than all) houses have a front door. Then functional anaphors based on such defining contexts still behave as we would want. In particular, the truth of (24) would guarantee that Cora knocked on the front door of $z$, even if there are more houses with a front door than are required to verify the weakened, generic version of (23). While for a $z$ that is not a house (e.g., if (22) had said "Cora walked up to a house or a trailer home", and only the latter alternative is true), $y(z)$ could have an arbitrary denotation that accidentally verifies (24). However, under our text semantics (17), such a misapplied reference would again just lead to falsity of the predication containing it, here (24).

\subsection{Related work and concluding comments}

The author's previous 'dynamic Skolemization' proposal [18] was aimed at the same kinds of applications as the present one, but involved syntactic substitution of constants/functions for existentials, accompanied by stipulation of definitional clauses for these constants/functions. The advantage of this strategy is that it directly yields ordinary firstorder sentences, avoiding dynamic semantics. Most instances of donkey anaphora and functional anaphora are amenable to it. However, as already noted, dynamic Skolemization is viable in unnegated contexts only; and extending it to negated contexts would require rather complex restructuring of the original logical forms. Thus the present approach is more general and theoretically satisfactory.

In [20], Steedman sketches an approach to donkey anaphora wherein an indefinite like " $a$ donkey" would receive an initial logical form like arb 'donkey', which would in turn yield a Skolem term like Skdonkey $(x), x$ being the universally quantified variable(s) in whose quantifier scopes the indefinite is included. This appears to be similar to dynamic Skolemization, though the syntactic details and mechanism weren't spelled out at the time. Steedman subsequently elaborated this idea in [21], essentially rendering an indefinite NP of form "an $N$ " as skolem $i\left(\lambda x N^{\prime}(x)\right)$, regarded as an underspecified term with unique index $i$, to be replaced by an ordinary Skolem function when the functional dependency of the individual 'arbitrarily selected' from the truth set of the predicate can be determined. However, the Skolem constants and functions are not treated in the semantics as ordinary constants and functions, but rather are dynamically interpreted, rather like DRT discourse referents or DPL $\exists$-variables, apart from the introduction of explicit functional dependencies. In that 
respect, Steedman's approach has similarities with both dynamic Skolemization (though the latter treats Skolem constants/functions as ordinary, nondynamic constants/functions), and implicit Skolemization (though the latter retains the $\exists$-quantified form of indefinites). Detailed comparisons are difficult at this point because of the extensive technical differences between the two approaches, and because different phenomena have been focused on (e.g., scope ambiguity in Steedman's work, and extension to quantifiers like Most in the present work).

The use of Skolem functions for indefinites has also been studied by a number of other researchers, e.g., Schlenker [16, 17], Winter [26], Barker [4], Hardt [11], and Dekker [7], often with a view towards sorting out the strengths and weaknesses of various approaches to the logic of E-type pronouns [8], scope ambiguity, and branching quantifiers. Besides Skolem function-based approaches, these approaches include choice functions and 'pronoun of laziness' accounts. The motivating examples in these discussions are often very similar to the ones that provided the impetus for the dynamic Skolemiztion proposal and for the present work. For example, Dekker [7] cites an example attributed to Gabriel Sandu,

(27) Most men had a gun, but only a few used it,

which clearly involves functional reference, and which lends itself nicely to the implicit Skolemization proposal herein. The same paper also cites a sentence due to Schlenker [16],

(28) If each student $x$ improves in two subjects $y$, then noone will fail the exam

[variables $x, y$ added here for convenience],

where the speaker has in mind not only that the two subjects vary from student to student, but that they are any given student's worst subjects. Again, this could be straightforwardly expressed in the current framework, with the speaker's presumption stated as

(29) For each student $x, y(x)$ are $x$ 's worst two subjects.

However, while the literature provides some nice examples motivating a Skolemized approach, the mechanism for Skolem function introduction has generally not been spelled out in detail, either as a systematic syntactic strategy, or as an implicit semantic phenomenon.

Two other kinds of theories of anaphora, formulated within DPL-like frameworks but with little resemblance to Skolemized approaches and emphasizing the interpretation of plural pronouns, have formally addressed some of the phenomena motivating implicit Skolemization. One kind of theory, exemplified by [12] and [25], models the dependency of variables on other variables by using augmented variable assignments whose values are individuals paired with variable assignments. For example, the dependence of $y$ on $x$ in "Every student $x$ wrote a paper $y$ " would be reflected in augmented assignments where the various possible individuals assigned to $x$ are paired with assignments that assign appropriate dependent values to $y$. The second kind of theory, exemplified by [22, 24, 23] and [14], relies simply on sets of (partial) variable assignments as input and output states of utterances. In this case the dependence of a variable $y$ on a variable $x$ is implicit in the fact that when we examine different assignments within the same state, we find that a particular value of $y$ co-occurs with some value of $x$, but not with some other value of $x$ (even though both values of $x$ occur in the same state). For simple plural reference, as in "The students $x$ were smart and the papers y were of high quality", these dependencies play no role - what matters is just the set of values assigned to $x$ or to $y$ by different assignments in the output state of the utterance that provides the plural referents for $x$ and $y$. But the dependencies can be exploited by use of a distribution operator that can be thought of as interpreting the English floating quantifier each. Thus "They $x$ each submitted it (their paper y) to LESP" is understood formally as involving a distribution operator that ensures that a given input state will generate a given output state only if for any given value $d$ of $x$, the subset of 
assignments of the input state that assign $d$ to $x$ can generate the subset of assignments in the output state that assign $d$ to $x .^{10}$

Both types of theories are aimed at pronominal anaphora, such as we find in

(30) Every man loves a woman. They send them flowers.

Dependencies in such examples are always 'short-range' - a pronoun cannot be used to refer to a distant linguistic antecedent, especially not if a functional dependency is involved. For example, we cannot paraphrase the second sentence in (22) as "She knocked on \#it", intending it to refer to a front door or front-door function introduced many utterances earlier and not mentioned subsequently. In the writings cited above, determining referents of anaphoric definite descriptions is viewed as an AI problem lying outside the immediate purview of dynamic semantics. Still, here is an attempt to use Nouwen's formalism [14] ${ }^{11}$ to deal with the second sentence in (22), assuming that a sentence like (23) (interpreting "Every house has a front door") has been introduced at some earlier point, setting up correlated variables $x$ (for houses) and $y$ (for front doors) in the variable assignments comprising the resultant state; assume that $x^{\prime}$ is the variable introduced for the house that Cora walked up to:

$$
\exists x^{\prime \prime} \dot{x}^{\prime \prime} \subseteq x \dot{x}^{\prime \prime}=x^{\prime} \dot{\delta}^{*}(\lambda u . \text { knock-on }(\text { Cora }, y))\left(x^{\prime \prime}\right) .
$$

This introduces a new variable $x^{\prime \prime}$, setting its value to the house $x^{\prime}$ under consideration (where the subset relation means that any (partial) variable assignment that supplies a value for $x^{\prime \prime}$ must supply the same value for $x$ ). The purpose of the new variable is to ensure that any assignment that provides a value for $x^{\prime \prime}$ will also provide the appropriate correlated value for $y$, i.e., the front door of $x^{\prime \prime}$ (and hence $x^{\prime}$, in view of the equality $x^{\prime \prime}=x^{\prime}$ ). This then makes it possible to apply the distribution operator $\delta^{*}$ to a predicate expressing the property of being an entity $u$ such that Cora knocks on a certain thing or set of things $y$. Though the $\lambda$-abstraction is vacuous, application of the distributed version of the predicate to $x^{\prime \prime}$ ensures that the only values of $y$ that can verify knock-on(Cora,y) are ones corresponding to possible values of $x^{\prime \prime}$, of which there is but one - the front door of the house $x^{\prime \prime}=x^{\prime}$.

We will not make any similar attempt to formulate a 'long-range' functional anaphor in the syntactically rather unconventional and semantically quite complex formalisms of [12] or [25]; even the above van den Berg / Nouwen-like representation may be remote from what those authors would propose. Obviously, this is a matter for further research and for the time being no definite conclusions can be drawn about the relative merits of these theories and the approach based on implicit Skolemization, with respect to the general phenomenon of functionally dependent anaphora. Implicit Skolemization does seem to have some advantages from a computational semantics perspective, in that the syntax of (restricted) quantification is conventional, and functional reference is syntactically simple and explicit and is semantically 'persistent' in the sense that implicitly established Skolem functions remain available for anaphoric reference even if their defining context lies in an arbitrarily large knowledge base (as mentioned in the discussion of bridging anaphora and frame- or script-like knowledge).

An interesting question raised by the cited work on plural anaphora, however, is whether

\footnotetext{
${ }^{10}$ It may appear from these remarks that the dynamics of meaning in the theories mentioned here depends on the names of variables used - as it does for existential variables in implicit Skolemization. While this is true for most of the theories, Nouwen's formalization is actually variable-free, and provides a basis for a bottom-up dynamic semantics for natural language that is compositional in the strong sense of not requiring a level of semantic representation.

${ }^{11}$ with some slight adjustments harking back to van den Berg [22], for expository reasons
} 
implicit Skolemization could be extended to deal systematically with plurals. For example, do we have a way of picking out the set of students and the set of papers they wrote, given that "Most students $x$ wrote a paper $y$ "? Here are some cursory observations. In the example, we could access the set of students who wrote papers and the set of papers that were written respectively as the domain and range of the implicit Skolem function, i.e., $\{c \mid(\exists d) d=y(c)\}$ and $\{d \mid(\exists c) d=y(c)\}$ (which of course could be reformulated in terms of set membership and quantification over sets, or in various other ways). ${ }^{12}$ However, what if no functional dependency is involved, as in "Most students stayed home; they felt the recitation was redundant"? One possibility is to introduce Davidsonian event variables into sentence predicates, which would then be implicitly Skolemized and could thus supply the required sets. Another possibility is to introduce existentially quantified set variables with all noun phrase interpretations, which is essentially the strategy in [25]. Various issues arise concerning accessibility of referents and the 'division of labor' between semantics and pragmatics (cf. [14, 25]), but these are matters for future investigation.

\section{Acknowledgements}

The author gratefully acknowledges the very perceptive comments of Ken Shan, Philippe Schlenker, Rick Nouwen, and the anonymous referees on the preliminary version [19] or current version of this paper. These comments led to quite significant revisions and (one hopes) improvements. As already mentioned, Ken Shan's comments were the inspiration for section 3.1; also the deployment of a partial-function semantics was his suggestion (though the implementation of that suggestion here may be remote from what he had in mind). Many thanks as well to the editor, Alastair Butler, for his patience and guidance. This work was supported in part by NSF grants IIS-0082928 and IIS-0328849.

\section{Appendix: Proofs}

We prepare for the proof of the Implicit Skolemization theorem by noting the following:

Lemma. Regardless of $\mathcal{M}$ and $\phi$, only $\exists$-variables can be $\mathcal{M}$-dynamic in $\phi$.

Proof. Assume an arbitrary model $\mathcal{M}$ and formula $\phi$. Variables not bound in $\phi$ can be seen to be uniformly static in $\phi$ by an easy induction, with atomic formulas as basis. Note that in the $\forall$-semantics in (6), any variable $y$ that is not bound in the scope will be static by the induction assumption, and so we will have $V(y)=U(y)$, i.e., the variable remains static. By the same token, we have $V(x)=U(x)$, where $x$ is the $\forall$-quantified variable in (6) (and is thus not bound within the scope of the quantifier), so variables that are $\forall$-quantified in a formula $\phi$ are also uniformly static. On the other hand, for the $\exists$-quantified variable $y$ in (5), $U$ may differ from $U_{x: d}$ at $x$, and thus the induction argument showing that variables that are static in smaller formulas are also static in larger ones fails for $\exists$-quantified variables, and only for these.

Theorem (Implicit Skolemization). For any formula $\phi$ and model $\mathcal{M}$, if $\langle U, V\rangle \in \llbracket \phi]]_{\mathcal{M}}$ then for any variable $y$ that is $\mathcal{M}$-dynamic in $\phi$, $\phi$ contains a (unique) $\exists$-quantifier binding $y$, where that quantifier is not embedded by any negation in $\phi$, and $V(y) \in \mathcal{F}_{n}$ where $n$ is the number of $\forall$-quantifiers in $\phi$ embedding the $\exists$-quantifier for $y$.

Proof. (a) Let $\phi$ be any formula, let $\mathcal{M}$ be any model, let $\langle U, V\rangle \in \llbracket \phi]_{\mathcal{M}}$, and assume that $y$ is $\mathcal{M}$-dynamic in $\phi$. Then by the lemma, $y$ is $\exists$-quantified in $\phi$. Its quantifier cannot

\footnotetext{
${ }^{12}$ The way we have formulated the partial function semantics would ensure that the domain will be the set of all students who wrote a paper, and the range will contain one paper for each of these students.
} 
be embedded by a negation, since the semantics of negation renders any variable $y$ in its scope $\mathcal{M}$-static, and this property is inherited by all larger formulas embedding the negation except possibly a larger formula that $\exists$-quantifies $y$ (by the same sort of inductive argument that was sketched in the proof of the lemma) - but according to our assumed syntax a variable cannot be $\exists$-quantified at a 'higher' level, when it is already $\exists$-quantified within the scope of the higher-level quantifier.

It remains to show that $V(y) \in \mathcal{F}_{n}$ where $n$ is the number of $\forall$-quantifiers in $\phi$ embedding the $\exists$-quantifier for $y$. As a basis for induction, consider the case $\phi=(\exists y) \psi$. By (5), and since $y$ is static in $\psi, V(y)=U_{y: d}(y)=d$ for some $d \in \mathcal{D}$, and so $V(y) \in \mathcal{F}_{0}$. The induction hypothesis is that the theorem holds for all formulas of a certain size $s$ or less. We now consider the cases where $\phi$ is $\left(\chi \wedge \chi^{\prime}\right),\left(\chi^{\prime} \wedge \chi\right),\left(\chi \vee \chi^{\prime}\right),\left(\chi^{\prime} \vee \chi\right),\left(\chi \rightarrow \chi^{\prime}\right),\left(\chi^{\prime} \rightarrow \chi\right)$, $(\exists x) \chi$, or $(\forall x) \chi$, where $\chi, \chi^{\prime}$ are of size $s$ or less, $\chi$ contains $(\exists y) \psi$, and $\chi^{\prime}$ does not contain an $\exists$-quantified occurrence of $y$ (and no occurrence of $y$ at all in the three cases where $\chi^{\prime}$ precedes $\chi$ ). (Note that we are omitting $\phi=\neg \chi$, as this form is inconsistent with the assumption that $y$ is $\mathcal{M}$-dynamic in $\phi$.) We show in each case that $V(y) \in \mathcal{F}_{n}$, with $n$ as stipulated above.

$\left(\chi \wedge \chi^{\prime}\right)$ : According to the semantics of conjunction, there is a gva $U^{\prime}$ such that $\left\langle U, U^{\prime}\right\rangle$ $\in \llbracket \chi \rrbracket_{\mathcal{M}}$, and $\left.\left\langle U^{\prime}, V\right\rangle \in \llbracket \chi^{\prime}\right]_{\mathcal{M}}$. Since $y$ is assumed to be dynamic in $\phi$, and since it is $\exists$-quantified in $\chi$, the induction hypothesis implies that $U^{\prime}(y) \in \mathcal{F}_{k}$, where $k$ is the number of $\forall$-quantifiers embedding $(\exists y) \psi$ in $\chi$ (for some $\psi$ ). But $y$ is not $\exists$-quantified in $\chi^{\prime}$, so it is static in $\chi^{\prime}$, and so $V(y)=U^{\prime}(y)$ and hence $V(y) \in \mathcal{F}_{k}$, and of course $n=k$ here.

$\left(\chi^{\prime} \wedge \chi\right),\left(\chi \vee \chi^{\prime}\right),\left(\chi^{\prime} \vee \chi\right)$ : The argument for each of these cases is quite similar to the preceding one.

$\left(\chi \rightarrow \chi^{\prime}\right)$ : Here the assumption that $y$ is $\mathcal{M}$-dynamic in $\phi$ entails the first clause in (8), concerned with the case of a false antecedent (relative to $U$ ) does not apply. The second clause in (8) is essentially the semantics of conjunction, and so the same argument as for $\left(\chi \wedge \chi^{\prime}\right)$ applies.

$\left(\chi^{\prime} \rightarrow \chi\right)$ : Much the same argument as for $\left(\chi \rightarrow \chi^{\prime}\right)$ applies.

$(\exists x) \chi$ : Since $y$ is $\exists$-quantified in $\chi$, hence by our syntax $x$ and $y$ are distinct variables, and so $y$ must be $\mathcal{M}$-dynamic in $\chi$ (if it were $\mathcal{M}$-static in $\chi$ it would also be $\mathcal{M}$-static in $\phi$ ), and the induction hypothesis applies to $\chi$. Now, according to the $\exists$-semantics in (5), any pair $\langle U, V\rangle$ satisfying $(\exists x) \chi$ differs from a corresponding pair $\left\langle U_{x: d}, V\right\rangle$ satisfying $\chi$ at most in the value assigned by $U$ to $x$; so the value of $V(y)$ is inherited by $\phi$ from the scope $\chi$, and this is $\in \mathcal{F}_{k}$ by the induction hypothesis, where $k$ is the number of $\forall$-quantifiers embedding the $\exists$-quantifier of $y$ in $\chi$; and of course $n=k$.

$(\forall x) \chi$ : Again, since $y$ is $\mathcal{M}$-dynamic in $\phi$, it is $\mathcal{M}$-dynamic in $\chi$. (If $y$ were $\mathcal{M}$-static in $\chi$, it would be $\mathcal{M}$-static in $\phi$, by the condition for $\mathcal{M}$-static variables in (6).) So the induction hypothesis applies to $y$ in $\chi$. Hence in the condition derived from (6), that for all $d \in \mathcal{D}$, $\left.\left\langle U_{x: d}, V_{x: d}^{\prime}\right\rangle \in \llbracket \chi\right]_{\mathcal{M}}$, we know that $V_{x: d}^{\prime}(y) \in \mathcal{F}_{k}$, where $k$ is the number of $\forall$-quantifiers embedding the $\exists$-quantifier for $y$ in $\chi$. But (6) also posits that for $y \mathcal{M}$-dynamic in $\chi$, $V^{\prime}(y)=V(y)(d)$. Thus $V(y)$ is a function which, when applied to any $d \in \mathcal{D}$, yields a function in $\mathcal{F}_{k}$, provided that $V^{\prime}(y)$ is defined. In other words, $V(y) \in \mathcal{F}_{k+1}$, and of course $k+1=n$, the number of $\forall$-quantifiers embedding the $\exists$-quantifier for $y$ in $\phi(=(\forall x) \chi)$.

Proposition 1. For all models $\left.\mathcal{M}, \llbracket(\forall x: \phi) \psi]_{\mathcal{M}}=\llbracket(\forall x)(\phi \rightarrow \psi)\right]_{\mathcal{M}}$.

Proof. $\Leftarrow$ : Let $\langle U, V\rangle$ be in $\llbracket(\forall x)(\phi \rightarrow \psi)]_{\mathcal{M}}$. From (6) for all $d \in \mathcal{D}$, there is a gva $V^{\prime}$ such that $\left\langle U_{x: d}, V_{x: d}^{\prime}\right\rangle \in \llbracket \phi \rightarrow \psi \rrbracket_{\mathcal{M}}$, where for all variables $y$,

$V^{\prime}(y)=V(y)=U(y)$ if $y$ is $\mathcal{M}$-static in $(\phi \rightarrow \psi)$, and 


\section{$V^{\prime}(y)=V(y)(d)$ if $y$ is $\mathcal{M}$-dynamic in $(\phi \rightarrow \psi)$.}

Consider any $d \in \mathcal{D}$ and the corresponding $V^{\prime}$. First suppose $T_{\mathcal{M}, U}(x, \phi)=\emptyset$. Then $\left.\left\langle U_{x: d}, V_{x: d}^{\prime}\right\rangle \in \llbracket \phi \rightarrow \psi\right]_{\mathcal{M}}$ implies that $U_{x: d}=V_{x: d}^{\prime}$, by (8). But $x$ is static in $(\phi \rightarrow \psi)$, so $V^{\prime}(x)=U(x)$ and so $V^{\prime}=U$. In fact, with $T_{\mathcal{M}, U}(x, \phi)=\emptyset$, (8) implies that all variables are $\mathcal{M}$-static in $(\phi \rightarrow \psi)$, so $V^{\prime}=V=U$. This confirms case (a) of the $(\forall x: \phi) \psi$-semantics.

Now suppose $T_{\mathcal{M}, U}(x, \phi) \neq \emptyset$. Then from the premise that $\left.\left\langle U_{x: d}, V_{x: d}^{\prime}\right\rangle \in \llbracket \phi \rightarrow \psi\right]_{\mathcal{M}}$ and the further assumption that $d \in T_{\mathcal{M}, U}(x, \phi)$, it follows that $\left.\left\langle U_{x: d}, V_{x: d}^{\prime}\right\rangle \in \llbracket \phi \wedge \psi\right]_{\mathcal{M}}$. If that further assumption is false, $\left\langle U_{x: d}, V_{x: d}^{\prime}\right\rangle$ can be an element of $\left.\llbracket \phi \rightarrow \psi\right]_{\mathcal{M}}$ only if there is no $U^{\prime}$ such that $\left.\left\langle U_{x: d}, U^{\prime}\right\rangle \in \llbracket \phi\right]_{\mathcal{M}}$, by (8); in that case $V_{x: d}^{\prime}=U_{x: d}$, and thus $V^{\prime}=U$, since $x$ is static in $(\phi \rightarrow \psi)$. In either case, noting that a variable $y$ is $\mathcal{M}$-static [-dynamic] in $(\phi \wedge \psi)$ iff it is $\mathcal{M}$-static [-dynamic] in $(\phi \rightarrow \psi)$ (as is easily verified, since with $\llbracket \phi]_{\mathcal{M}}=\emptyset$, every variable is $\mathcal{M}$-static in $(\phi \wedge \psi))$,

$$
\begin{aligned}
& V^{\prime}(y)=V(y)=U(y) \text { if } y \text { is } \mathcal{M} \text {-static in }(\phi \wedge \psi), \text { and } \\
& V^{\prime}(y)=V(y)(d) \text { if } y \text { is } \mathcal{M} \text {-dynamic in }(\phi \wedge \psi) .
\end{aligned}
$$

This confirms case (b) of the $(\forall x: \phi) \psi$-semantics.

$\Rightarrow$ : Conversely, let $\langle U, V\rangle$ be in $\llbracket(\forall x: \phi) \psi]_{\mathcal{M}}$. Then from (19), either (a) $T_{\mathcal{M}, U}(x, \phi)=\emptyset$ and $V=U$, or (b) $T_{\mathcal{M}, U}(x, \phi) \neq \emptyset$ and for all $d \in \mathcal{D}$ there is a gva $V^{\prime}$ such that (i) if $d \in T_{\mathcal{M}, U}(x, \phi)$, then $\left.\left\langle U_{x: d}, V_{x: d}^{\prime}\right\rangle \in \llbracket \phi \wedge \psi\right]_{\mathcal{M}}$, and otherwise $V^{\prime}=U$; and (ii) for all variables $y$,

$$
\begin{aligned}
& V^{\prime}(y)=V(y)=U(y) \text { if } y \text { is } \mathcal{M} \text {-static in }(\phi \wedge \psi), \text { and } \\
& V^{\prime}(y)=V(y)(d) \text { if } y \text { is } \mathcal{M} \text {-dynamic in }(\phi \wedge \psi) .
\end{aligned}
$$

We want to show that $\langle U, V\rangle$ be in $\llbracket(\forall x)(\phi \rightarrow \psi)]_{\mathcal{M}}$, i.e., for all $d \in \mathcal{D}$, there is a gva $V^{\prime}$ such that $\left\langle U_{x: d}, V_{x: d}^{\prime}\right\rangle \in \llbracket \phi \rightarrow \psi \rrbracket_{\mathcal{M}}$, where for all variables $y, V^{\prime}(y)=V(y)=U(y)$ if $y$ is $\mathcal{M}$-static in $(\phi \rightarrow \psi)$, and $V^{\prime}(y)=V(y)(d)$ if $y$ is $\mathcal{M}$-dynamic in $(\phi \rightarrow \psi)$.

For case (a), $T_{\mathcal{M}, U}(x, \phi)=\emptyset$, so for any $\left.d \in \mathcal{D},\left\langle U_{x: d}, U_{x: d}\right\rangle \in \llbracket \phi \rightarrow \psi\right]_{\mathcal{M}}$ by (8), and so $U$ provides the required $V^{\prime}$ in the preceding statement. For case (b), where $T_{\mathcal{M}, U}(x, \phi) \neq \emptyset$, consider any $d \in \mathcal{D}$ and the corresponding $V^{\prime}$ assured by (b). Then if $d \in T_{\mathcal{M}, U}(x, \phi)$, we have $\left\langle U_{x: d}, V_{x: d}^{\prime}\right\rangle \in \llbracket \phi \wedge \psi \rrbracket_{\mathcal{M}}$, hence by (8), $\left\langle U_{x: d}, V_{x: d}^{\prime}\right\rangle \in \llbracket \phi \rightarrow \psi \rrbracket_{\mathcal{M}}$, and by (ii), and the previously noted equivalence of $(\phi \wedge \psi)$ and $(\phi \rightarrow \psi)$ with respect to the $\mathcal{M}$-static $/ \mathcal{M}$ dynamic distinction for any variable $y$,

$V^{\prime}(y)=V(y)=U(y)$ if $y$ is $\mathcal{M}$-static in $(\phi \rightarrow \psi)$, and

$V^{\prime}(y)=V(y)(d)$ if $y$ is $\mathcal{M}$-dynamic in $(\phi \rightarrow \psi)$.

If $d \notin T_{\mathcal{M}, U}(x, \phi)$, then with $V^{\prime}=U,\left\langle U_{x: d}, V_{x: d}^{\prime}\right\rangle=\left\langle U_{x: d}, U_{x: d}\right\rangle$ and this is in $\left.\llbracket \phi \rightarrow \psi\right]_{\mathcal{M}}$ by (8). Also, we again have from (ii) that

$V^{\prime}(y)=V(y)=U(y)$ if $y$ is $\mathcal{M}$-static in $(\phi \rightarrow \psi)$, and

$V^{\prime}(y)=V(y)(d)$ if $y$ is $\mathcal{M}$-dynamic in $(\phi \rightarrow \psi)$,

where in this case $V^{\prime}(y)=U(y)$.

\section{References}

[1] D. D. Ahn. Presupposition incorporation in adverbial quantification. In Modeling and Using Context: Proc. of CONTEXT 2003, pages 1-14, Stanford, CA, June 23-25 2003.

[2] D. D. Ahn. The Role of Situations and Presuppositions in restricting Adverbial Quantification. PhD thesis, Dept. of Computer Science, Univ. of Rochester, Rochester, NY 14627-0226, 2004. 
[3] F. Baader, D. Calvanese, D. McGuinness, D. Nardi, and P. Patel-Schneider, editors. The Description Logic Handbook: Theory, Implementations, and Applications. Cambridge U. Press, Cambridge, England, 2003.

[4] C. Barker. Integrity: A syntactic constraint on quantificational scoping. In K. Megerdoomian and L. A. Bar-el, editors, WCCFL 20, pages 56-67, USC, Feb. 23-25 2001. Cascadilla Press.

[5] G. Carlson and F. Pelletier. The Generic Book. U. of Chicago Press, 1995.

[6] G. Chierchia. Dynamics of Meaning. U. of Chicago Press, 1995.

[7] P. Dekker. Pronouns in a pragmatic semantics. J. of Pragmatics (in press).

[8] G. Evans. Pronouns. Linguistic Inquiry, 11:337-362, 1980.

[9] B. Geurts. Donkey business. Linguistics and Philosophy, 25:129-156, 2002.

[10] J. Groenendijk and M. Stokhof. Dynamic predicate logic. Linguistics and Philosophy, 14:39-100, 1991.

[11] D. Hardt. DRT and related theories, lecture IV: The E-type challenge. In ESSLLI 2003 class notes, 15th European Summer School in Logic Language and Information. Vienna University of Technology, Vienna, Austria, Aug.18-29 2003.

[12] M. Krifka. Parameterized sum individuals for plural anaphora. Linguistics and Philosophy, 19:555-598, 1996.

[13] H. Minsky. A framework for representing knowledge. In P. Winston, editor, The Psychology of Computer Vision, pages 211-277. McGraw-Hill, 1975.

[14] R. Nouwen. Plural pronominal anaphora in context: dynamic aspects of quantification. $\mathrm{PhD}$ thesis, UiL-OTS, Univ. of Utrecht, 2003.

[15] R. Schank and R. Abelson. Scripts, Plans, Goals and Understanding. Lawrence Erlbaum Assoc., Hillsdale, NJ, 1977.

[16] P. Schlenker. Skolem functions and the scope of indefinites. In P. Tamanji, editor, Proc. of the North-East Linguistics Society NELS 29), Amherst, MA, 1999.

[17] P. Schlenker. Scopal independence: On branching \& island-escaping readings of indefinites \& disjunctions. J. of Semantics, to appear, accepted 2004.

[18] L. Schubert. Dynamic Skolemization. In H. Bunt and R. Muskens, editors, Computing Meaning, vol. 1, pages 219-253. Kluwer Ac. Press, 1999.

[19] L. Schubert. Variables as functions: Efficient reference to dependent entities. In Workshop on Semantic Approaches to Binding Theory, 16th European Summer School in Logic, Language and Information (ESSLLI 2004), Universié Henri Poincaré, Nancy, Aug. 16-20 2004. Extended abstracts available at http://www.linguistics.ucla.edu/people/schlenker/ESSLLI04.html.

[20] M. Steedman. Alternating quantifier scope in CCG. In Proc. of 37th Ann. Meeting of the Assoc. for Computational Linguistics (ACL'99), pages 301-308, College Park, MD, June 20-26 1999. 
[21] M. Steedman. Scope alternation and the syntax-semantics interface. Abstract vailable at http://ling.northwestern.edu/ salt14/Abstracts/Steedman.pdf., 2003.

[22] M. H. van den Berg. Full dynamic plural logic. In K. Bimb'o and A. M'at'e, editors, Proc. of the 4th Symposium on Logic and Language, Budapest, 1993.

[23] M. H. van den Berg. Discourse grammar and dynamic logic. In Proc. of the 10th Amsterdam Colloquium, 1996.

[24] M. H. van den Berg. The Internal Structure of Discourse. PhD thesis, ILLC, Univ. of Amsterdam, 1996.

[25] L. Wang, E. McCready, and N. Asher. Information dependency in quantificational subordination. In Where Semantics Meets Pragmatics: 1st Int. Workshop on Current Research in the Semantics-Pragmatics Interface, Michigan State University, July 1113 2003. to appear in K. Turner \& K. von Heusinger (eds.), Where Semantics Meets Progmatics, Elsevier.

[26] Y. Winter. Choice functions and the scopal semantics of indefinites. Linguistics and Philosophy, 20:399-467, 1997. 